\title{
Evodiamine, a novel inhibitor of the Wnt pathway, inhibits the self-renewal of gastric cancer stem cells
}

\author{
ZHENZHEN WEN $^{1 *}$, SHUJIONG FENG ${ }^{2 *}$, LIJUAN WEI $^{3}$, ZHIMIN WANG $^{4}$, DEFEI HONG $^{4}$ and QIANG WANG ${ }^{4}$ \\ ${ }^{1}$ Department of Gastroenterology, Sir Run Run Shaw Hospital, School of Medicine, Zhejiang University, Hangzhou, \\ Zhejiang 310016; ${ }^{2}$ Department of Gastroenterology, Hangzhou Geriatric Hospital, The North Branch of Hangzhou \\ First People's Hospital Group, Hangzhou, Zhejiang 310012; ${ }^{3}$ Department of Traditional Chinese Medicine, Second Affiliated \\ Hospital of Zhejiang University School of Medicine, Hangzhou, Zhejiang 310009; ${ }^{4}$ Department of Hepatobiliary \\ and Pancreatic Surgery, Zhejiang Provincial People's Hospital, Hangzhou, Zhejiang 310014, P.R. China
}

Received April 7, 2015; Accepted October 8, 2015

DOI: $10.3892 / \mathrm{ijmm} .2015 .2383$

\begin{abstract}
Gastric cancer stem cells (GCSCs) have an important role in metastasis and recurrence of gastric cancer, and novel treatment strategies that target GCSCs are urgently required. Although evodiamine (Evo), a derivative of the traditional herbal medicine Evodia rutaecarpa, has been reported to have various biological effects, its effect on GCSCs remains unknown. In order to determine the effect of Evo on apoptosis of GCSCs, an MTS assay, flow cytometry and western blot analysis were performed. The effect of Evo on self-renewal in GCSCs was measured by alterations in the sphere formation ability, the expression of induced-pluripotent stem cell factors, expression of epithelial-to-mesenchymal transition (EMT) factors and oxaliplatin resistance of gastric cancer cells (GCCs). Evo inhibited proliferation, promoted the Bax/B-cell lymphoma 2 ratio and altered active caspase-3 expression of GCSCs. In addition, Evo decreased the sphere formation ability, the expression of Sox 2, KLF4, Bmi-1 and Oct4, and oxaliplatin resistance in GCCs. Evo decreased the expression of Slug, Twist, Zeb1 and vimentin, suggesting an inhibitory effect on EMT. Furthermore, the expression of $\beta$-catenin, c-Myc and cyclin D1 was decreased in Evo-treated spheroids from GCCs. In conclusion, Evo inhibited the Wnt/ $\beta$-catenin signaling pathway to inhibit proliferation and stem cell properties of GCSCs and repressed the EMT. The present findings highlight the prospect of Evo as a CSCs-targeted therapy in gastric cancer.
\end{abstract}

Correspondence to: Dr Qiang Wang, Department of Hepatobiliary and Pancreatic Surgery, Zhejiang Provincial People's Hospital, 158 Shang Tang Road, Hangzhou, Zhejiang 310014, P.R. China

E-mail: wazzbaby@163.com

*Contributed equally

Key words: evodiamine, self-renewal, gastric cancer stem cells, the Wnt/ $\beta$-catenin signaling pathway, epithelial-to-mesenchymal transition

\section{Introduction}

Gastric cancer is one of the most common types of cancer and the leading cause of cancer fatality worldwide (1). The overall prognosis is poor, with a 5 -year survival rate of $<40 \%$, primarily due to recurrence and distant metastasis (2). Recently, gastric cancer stem cells (GCSCs) were shown to promote tumorigenesis, aggressive growth, recurrence, metastasis and drug resistance, generating opportunities for the development of novel GCSC-targeted therapies in the treatment of gastric cancer $(3,4)$. Conventional anticancer treatments, such as surgery, systemic chemotherapy and ablative therapy, which can kill only differentiated cancer cells, result in tumor size reduction; however, they are often linked with gastric cancer relapses after some time. This relapse may be due to the presence of the residual GCSCs, demonstrating the requirement to develop drugs that specifically target cancer stem cells (CSCs).

GCSCs were first isolated and identified in 2009 and exhibit the stem cell properties self-renewal, multiple differentiation and strong tumorigenicity (5). Strong evidence has previously demonstrated that GCSCs are the major cause of invasion (6). The source of GCSCs remains unknown, although it has been postulated to originate from normal stem cells (SCs) that have undergone malignant transformation or progenitor cells that suffer oncogenic mutations and reactivation of stemness-related properties (4). Additionally, a previous study has indicated that epithelial-to-mesenchymal transition (EMT)-related proteins were associated with CSC-like properties in gastric cancer, which suggested that the EMT can generate GCSCs, although the role of this process remains a matter of debate (7). There are multiple signaling pathways that are essential for the maintenance of CSCs, including the Notch pathway, Hedgehog pathway, bone morphogenetic protein signaling, epidermal growth factor pathway and Wnt pathway (8-10). The Wnt pathways regulate cell growth, proliferation and survival, and abnormal activity of this pathway has been shown to promote self-renewal of CSCs, particularly in lung (11), breast (12) and colon cancer SCs (13). In addition, there is a strong correlation between Wnt1 and CD44 expression and the grade of gastric cancer. Stable overexpression of Wnt1 increased spheroid formation of adenocarcinoma gastric (AGS) cells and enriched the expression of Oct4 and CD44 (14). 
Also, Cai and Zhu (15) demonstrated that the Wnt/ $\beta$-catenin pathway is essential for the self-renewal of cancer stem-like cells in human gastric cancer by detecting the expression levels of $\beta$-catenin, c-myc, cyclin D1 and axin 2 in GCSCs. Therefore, given the important role of the Wnt pathway in GCSCs, further understanding of the regulation of Wnt pathways represents a viable therapeutic approach to target GCSCs.

Evodiamine (Evo) is a natural chemical derived from the plant Evodia rutaecarpa.Previously, Evo was shown to have biological effects, including antitumor, antinociceptive and vasorelaxant properties $(16,17)$. The strong antitumor effects of Evo occur via different mechanisms in a variety of tumors. In colon cancer, Evo activated c-Jun N-terminal kinase, leading to subsequent activation of apoptosis and G2/M arrest (18). Evo may inhibit transforming growth factor- $\beta 1$-induced EMT in NRK52E cells via the Smad and peroxisome proliferator-activated receptor- $\gamma$ pathway (19). Additionally, Evo inhibited growth and induced apoptosis and autophagy in gastric cancer (20). Thus far, the effect of Evo on GCSCs remains unclear.

In the present study, the effects of Evo on the viability of GCSCs and signaling pathways regulating apoptosis and self-renewal in GCSCs were investigated. These findings provide a new option for the treatment of gastric cancer by specifically targeting GCSCs.

\section{Materials and methods}

Cell lines and culture. Gastric cancer cell lines AGS and SGC7901 were purchased from the Cell Bank of the Shanghai Branch of the Chinese Academy of Sciences, Shanghai Institute of Cell Biology (Shanghai, China). The gastric cancer cell lines were cultured in RPMI-1640 medium (Gibco, Thermo Fisher Scientific, Grand Island, NY, USA) supplemented with $100 \mathrm{U} / \mathrm{ml}$ penicillin, $100 \mu \mathrm{g} / \mathrm{ml}$ streptomycin and $10 \%$ fetal bovine serum, and maintained at $37^{\circ} \mathrm{C}$ in a $5 \% \mathrm{CO}_{2}$ humid atmosphere. Cancer stem cells were enriched from these cells at $1 \times 10^{4}$ cells/well in serum-free medium [ $20 \mu \mathrm{g} / \mathrm{l}$ epidermal growth factor (Invitrogen, Carlsbad, CA, USA)], 1X B27 (Invitrogen), $20 \mu \mathrm{g} / \mathrm{l}$ basic fibroblast growth factor (Invitrogen), $0.4 \%$ bovine serum albumin (Roche, Mannhein, Germany), $4 \mathrm{mg} / \mathrm{l}$ insulin (Invitrogen) and $200 \mathrm{IU} / \mathrm{ml}$ penicillin/streptomycin (Sangon Biotech Co., Ltd., Shanghai, China) on poly-HEMA-coated 6-well plates (Corning, New York, NY, USA).

Reagents. Evo (Sigma-Aldrich, St. Louis, MO, USA) was dissolved in dimethylsulfoxide (DMSO; Sigma-Aldrich) to generate a 30-mM stock solution and diluted in RPMI-1640 medium (Gibco) prior to use. The final concentration of DMSO in all cell culture was $<0.5 \%$ and did not have any harmful effects on cell growth.

3-(4,5-dimethylthiazol-2-yl)-5-(3-carboxymethoxyphenyl)2-(4-sulfophenyl)-2H-tetrazolium (MTS) was purchased from Promega (Madison, WI, USA). The Annexin V-FITC reagent and propidium iodide (PI) were supplied by BD Biosciences (San Jose, CA, USA), and the $\beta$-catenin/Tcf inhibitor was purchased from Merck (Merck KGaA, Darmstadt, Germany).

MTS assay. CSCs enriched from AGS and SGC7901 cells were dispensed (100 $\mu \mathrm{l}$ medium/well) in triplicate into a 96-well plate at a density of $5 \times 10^{3}$ cells. Cells were treated with $(1,2,4$ and
$8 \mu \mathrm{M}$ ) or without Evo for 24, 48 and $72 \mathrm{~h}$. Subsequently, $20 \mu \mathrm{l}$ MTS was added to each well and incubated for $4 \mathrm{~h}$. Absorbance was measured at $490 \mathrm{~nm}$ using a Thermo Varioskan Flash Reader (Thermo Fisher Scientific, Cambridge, MA, USA). The $\mathrm{IC}_{50}$ values for 24,48 and $72 \mathrm{~h}$ were calculated using the SPSS 13.0 software (SPSS, Inc., Chicago, IL, USA).

Flow cytometry. Enriched CSCs treated with or without Evo $(2 \mu \mathrm{M})$ for $48 \mathrm{~h}$ were digested and harvested in $1 \mathrm{X}$ binding buffer (0.1 M Hepes, $1.4 \mathrm{M} \mathrm{NaCl}$ and $\left.25 \mathrm{mM} \mathrm{CaCl}_{2}\right)$. To the solution ( $1 \times 10^{5}$ cells), $5 \mu \mathrm{l}$ of Annexin $\mathrm{V}$ and $5 \mu \mathrm{l}$ of PI were added, and subsequently incubated at room temperature in the dark for $15 \mathrm{~min}$. Binding buffer $(400 \mu \mathrm{l})$ was added to each tube and samples were subjected to flow cytometric analysis (BD Biosciences) within $1 \mathrm{~h}$.

Western blot analysis. AGS and SGC7901 cells and CSCs enriched from them were harvested and lysed in $350 \mathrm{ml}$ radioimmunoprecipitation assay buffer.Proteins were resolved using $12 \%$ sodium dodecyl sulfate-polyacrylamide gel electrophoresis and electrophoretically transferred onto nitrocellulose membranes. Membranes were incubated overnight at $4^{\circ} \mathrm{C}$ with the following primary monoclonal antibodies: B-cell lymphoma 2 (Bcl-2; ab32124; rabbit monoclonal to human), Bax (ab7977; rabbit polyclonal to human), caspase-3 (ab2171; mouse monoclonal to human), $\beta$-catenin (ab6302; rabbit polyclonal to human), c-Myc (ab32072; rabbit monoclonal to human), cyclin D1 (ab16663; rabbit monoclonal to human), E-cadherin (ab1416; mouse monoclonal to human), vimentin (ab133260; rabbit monoclonal to human), Slug (ab27568; rabbit polyclonal to human), Twist (ab50581; rabbit polyclonal to human; 1:1,000-2,000; Epitomics, Burlingame, CA, USA), Bmi1 (ab38295; rabbit polyclonal to human), Sox 2 (ab97959; rabbit polyclonal to human), Oct4 (ab18976; rabbit polyclonal to human), kruppel-like factor (KLF)4 (ab72543; rabbit polyclonal to human; 1:500-1:1,000; Abcam, Cambridge, UK), and glyceraldehyde-3-phosphate dehydrogenase (GAPDH; KC-5G4; mouse monoclonal to human) (1:5,000; Kangcheng Biology Engineering Co., Ltd., Shanghai, China). After five washes, membranes were incubated with a horseradish peroxidase-labeled goat anti-rabbit polyclonal antibody (1:2;000; Jackson ImmunoResearch Laboratory, West Grove, PA, USA) at room temperature for $1 \mathrm{~h}$. The reactive bands were detected using enhanced chemiluminescence (Cell Signaling Technology, Beverley, MA, USA).

Sphere formation assay. AGS and SGC7901 cells were seeded in poly-HEMA-coated 24 -well plates (150 cells/well) with serum-free medium, followed by incubation at $37^{\circ} \mathrm{C}$ in a $5 \%$ $\mathrm{CO}_{2}$ atmosphere for 5-7 days. Sphere formation efficiency was calculated as the number of spheres/cell number x $100 \%$.

Reverse transcription-quantitative polymerase chain reaction (RT-qPCR). Detection of mature ribonucleic acids (RNAs) was performed using the TaqMan RT-qPCR method (Applied Biosystems, Foster City, CA, USA) according to the manufacturer's protocol. Total RNA was isolated from AGS and SGC7901 cells using the Rneasy Mini kit (Qiagen, Hilden, Germany); reverse transcription was performed following quantitation using PrimeScript RT reagent kit with gDNA Eraser (Takara, Shiga, Japan). The reverse transcription 
products were mixed with specific primers and probes, and RT-qPCR was performed. The PCR program was as follows: $95^{\circ} \mathrm{C}$ for $30 \mathrm{sec}$, followed by 40 cycles of $5 \mathrm{sec}$ at $95^{\circ} \mathrm{C}$ and $34 \mathrm{sec}$ at $60^{\circ} \mathrm{C}$. The primers and probe for Sox 2 were as follows: Top, 5'-aatgecttcatggtgtgg-3' and bottom, 5'-cttctccgtctccgacaa a-3'; and probe, 5'Fam-agtttccactcggcgeccag-3'Tamra. The primers and probe for KLF4 were as follows: Top, 5'-ggcact accgtaaacacacg-3' and bottom, 5'-ctggcagtgtgggtcatatc-3'; and probe: 5'Fam-caggtcggaccacctcgect-3'Tamra. The primers and probe for Oct4 were as follows: Top, 5'-gtggaggaagctgac aacaa-3' and bottom, 5'-aacaaattctccaggttgcc-3'; and probe: 5'Fam-tctctttcgggcetgcacga-3'Tamra. Relative quantification of RNA levels was normalized to GAPDH and presented using the $\Delta \Delta \mathrm{Ct}$ method.

Drug resistance assay. AGS and SGC7901 cells were seeded in a 24 -well plate at a density of $1 \times 10^{4}$ cells/well in medium contained oxaliplatin (1.5 $\mu \mathrm{g} / \mathrm{ml}$ ) (Jiangsu Hengrui Medicine Co., Ltd., Jiangsu, China) or oxaliplatin and Evo $(2 \mu \mathrm{M} / 4 \mu \mathrm{M}$, respectively). The total number of viable AGS and SGC7901 cells were counted on days 3,5 and 7 .

Wound-healing assay. AGS and SGC7901 cells were grown to confluence in 6-cm dishes and wounds were generated using P-200 pipette tips. Cells were treated with or without Evo $(2 \mu \mathrm{M})$ for $48 \mathrm{~h}$. Phase contrast images were captured at 0 and $48 \mathrm{~h}$.

Statistical analysis. All the data are presented as mean \pm standard deviation (SD) of triplicate experiments and were analyzed with GraphPad Prism 5 (GraphPad Software, La Jolla, CA, USA) and SPSS software, version 13.0. Data were checked for normality and equal variances and transformed when necessary to meet the assumption of normal distribution. Comparisons between the two groups were performed using independent sample t-test. Differences between multiple groups were determined using one-way analysis of variance (ANOVA) with least significant difference test or Tamhane's T2 post hoc test. $\mathrm{P}<0.05$ was considered to indicate a statistically significant difference.

\section{Results}

Evo inhibits proliferation of GCSCs. The structure of Evo is shown in Fig. 1. GCSCs were enriched from AGS (Fig. 2A) and SGC7901 cells (Fig. 2B), respectively, in low-attachment plates with serum-free medium. In order to determine the effect of Evo on cell proliferation, an MTS assay was performed. Cell viability was significantly decreased in Evo-treated cells in a dose- and time-dependent manner (Fig. 3). When the Evo concentration reached $2 \mu \mathrm{mol} / 1$, the cell survival rates were significantly decreased by $24.78 \pm 2.1,33.73 \pm 2.91$ and $54 \pm 1.98 \%$ in AGS, and $35.91 \pm 2.68,41.78 \pm 3.41$ and $45.76 \pm 2.38 \%$ in SGC7901 cells (mean $\pm \mathrm{SD}, \mathrm{n}=9, \mathrm{P}<0.05$ ) at 24,48 and $72 \mathrm{~h}$, respectively. The $\mathrm{IC}_{50}$ values of Evo on GCSCs at 24, 48 and $72 \mathrm{~h}$ are shown in Table I. The results showed that evodiamine inhibited the proliferation of GCSCs in a dose- and time-dependent manner.

Evo induces apoptosis in GCSCs. Flow cytometric analysis was performed to determine the mechanism of Evo-mediated apotosis in GCSCs, and the percentage of Annexin V-positive GCSCs was significantly increased by Evo. Evo $(2 \mu \mathrm{M}, 48 \mathrm{~h})$
Table I. $\mathrm{IC}_{50}$ of evodiamine on colonspheres enriched from AGS and SGC7901 cells.

\begin{tabular}{|c|c|c|c|}
\hline $\mathrm{IC}_{50}$ & $24 \mathrm{~h}$ & $48 \mathrm{~h}$ & $72 \mathrm{~h}$ \\
\hline $\mathrm{AGS}, \mu \mathrm{M}$ & 5.06 & 3.14 & 1.92 \\
\hline 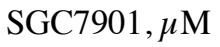 & 3.54 & 2.31 & 1.91 \\
\hline
\end{tabular}

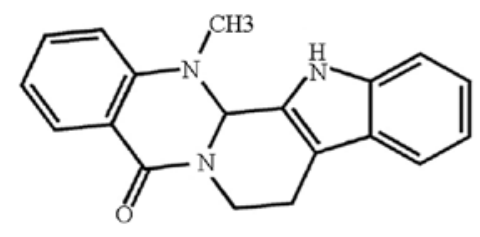

Figure 1. Chemical structure of evodiamine.

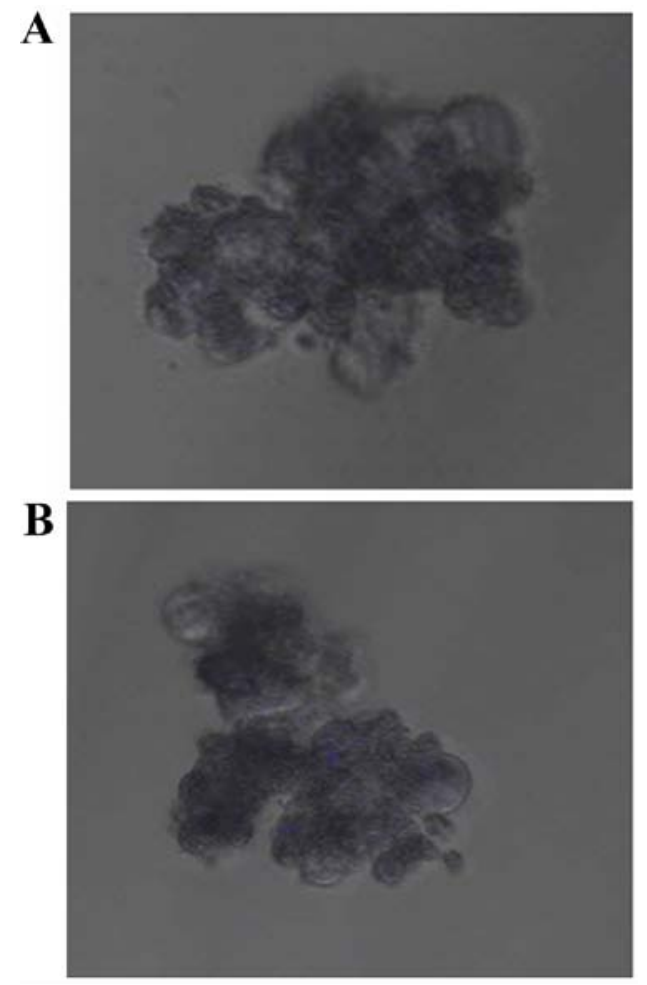

Figure 2. Colospheres enriched from (A) AGS and (B) SGC7901 cells.

increased rates of apoptosis from 11.6 to $23.9 \%$ and 5.2 to $11.2 \%$ in GCSCs from AGS and SGC7901 cells, respectively (Fig. 4A and B). These results were confirmed with western blot analysis. Evo elevated the Bcl-2 family members, Bax and active caspase-3, and reduced Bcl-2 in GCSCs (Fig. 4C and D). These findings suggested that Evo activated caspase-3-dependent apoptosis in GCSCs.

Evo inhibits the self-renewal of GCSCs. Sphere formation efficiency, a measure of self-renewal, was significantly decreased in the AGS and SGC7901 cells treated with Evo (Fig. 5A). Subsequently, the effect of Evo on drug resistance in gastric cancer cells (GCCs) was examined. Combination treatment with Evo and oxaliplatin significantly reduced the 
A

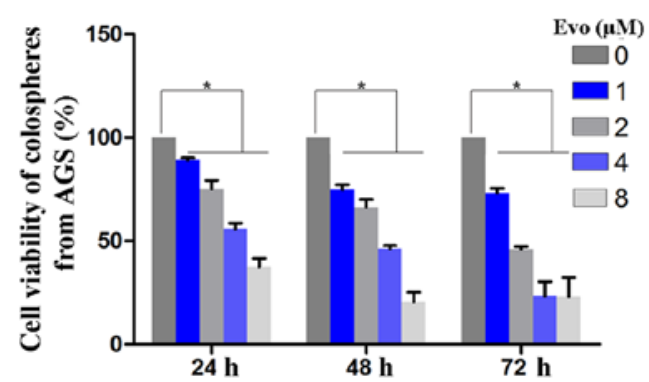

B

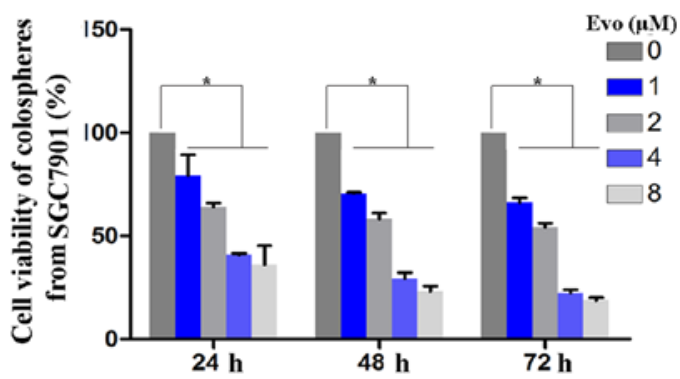

Figure 3. Evo inhibits the proliferation of GCSCs from AGS and SGC7901 cells in a dose- and time-dependent manner. The cell viability of GCSCs enriched from (A) AGS and (B) SGC7901 cells was assessed and expressed as percentage of the control. Results are presented as mean \pm standard deviation. Data were extracted from three independent experiments. ( $\mathrm{P}<0.05$, analysis of variance). GCSCs, gastric cancel stem cells.
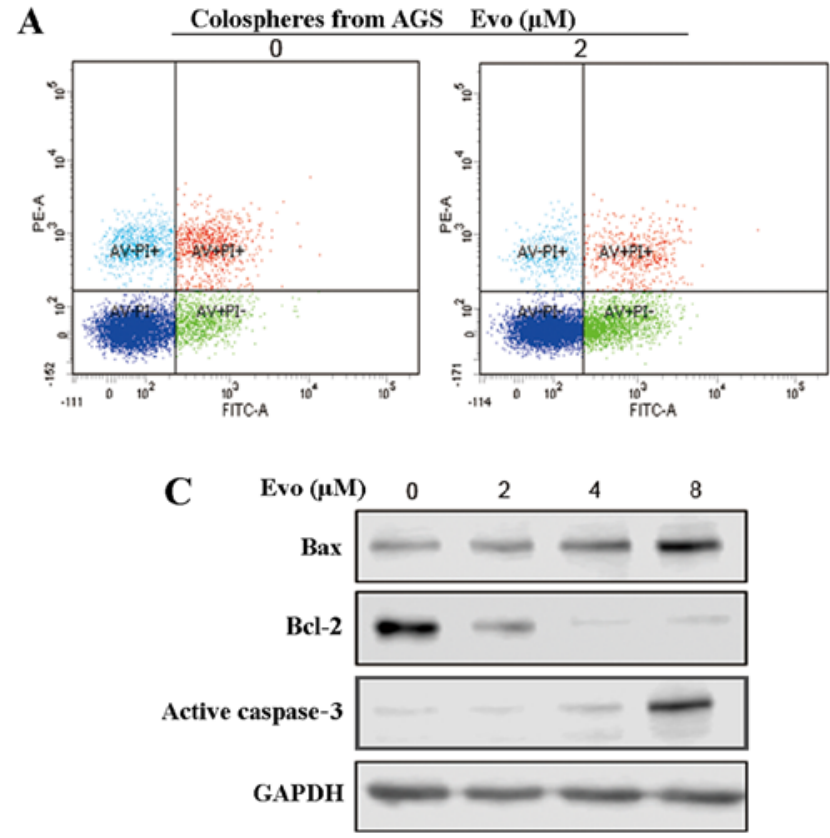

B
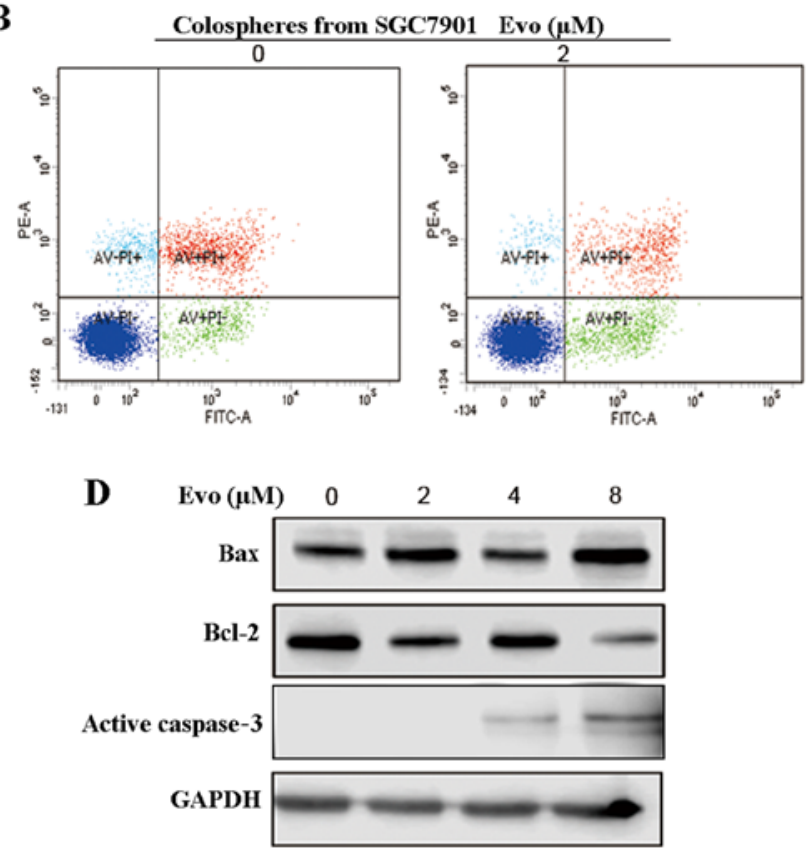

Figure 4. Evo induces apoptosis of GCSCs from AGS and SGC7901 cells in a dose-dependent manner. (A and B) Evo induces apoptosis in GCSCs. GCSCs enriched from (A) AGS and (B) SGC7901 cells were treated with Evo and harvested for detection of Annexin V-positive cells by flow cytometric analysis. (C and D) The effects of Evo on antiapoptotic Bcl-2 family member and caspase-3. GCSCs enriched from (C) AGS and (D) SGC7901 were treated with Evo at the indicated concentrations for $48 \mathrm{~h}$ and were subsequently harvested for western blotting to detect the expression of Bax, Bcl-2 and active caspase-3. GCSCs, gastric cancel stem cells; Bcl-2, B-cell lymphoma 2.

cell viability in a dose-dependent manner relative to control and oxaliplatin alone in AGS (Fig. 5B) and SGC7901 (Fig. 5C) cells. Finally, RT-qPCR was performed to detect the expression levels of induced-pluripotent stem (iPS) cell factors. The levels of Sox2, KLF4 and Oct4 were all increased in GCSCs treated with Evo relative to control ( $\mathrm{P}<0.05$, ANOVA) (Fig. 5D). Similarly, western blot analysis revealed that Evo treatment decreased the expression of iPS factors in AGS (Fig. 5E) and SGC7901 (Fig. 5F) cells. Taken together, these data indicated that Evo inhibited the self-renewal of GCSCs.

Evo confers resistance of EMT in GCCs. As Evo inhibited anoikis and self-renewal of GCSCs, and recent studies confirmed that the EMT is involved in resistance to anoikis and generation of CSCs, it follows that Evo may induce apoptosis and reduce stemness of GCCs by affecting the EMT process. Therefore, the effect of Evo on the migratory properties of GCCs was examined. The wound-healing migration assay showed that the wound-healing ability of AGS and SGC7901 cells treated with Evo ( $2 \mu \mathrm{M}$ for $48 \mathrm{~h}$ ) was significantly decreased relative to untreated controls (Fig. 6A and B). Concordant with the aforementioned observations, Evo reduced the expression of relevant EMT markers in GCCs. Western blot analysis showed that Slug, Twist, Zeb1 and vimentin were reduced with Evo treatment (Fig. 6C). In summary, Evo repressed EMT in GCCs.

Evo-mediated inhibition of GCSCs is through the Wnt/ $\beta$-catenin signaling pathway. The aforementioned experiments demonstrated a clear effect of Evo on GCSCs; however, the molecular mechanism was unclear. Abnormal activation of the Wnt pathway has been described in CSCs, therefore Evo-mediated inhibition of GCSCs is possibly through the Wnt pathway. Using western blot analysis, downstream molecules of Wnt, including c-Myc and cyclin D1, were 
A
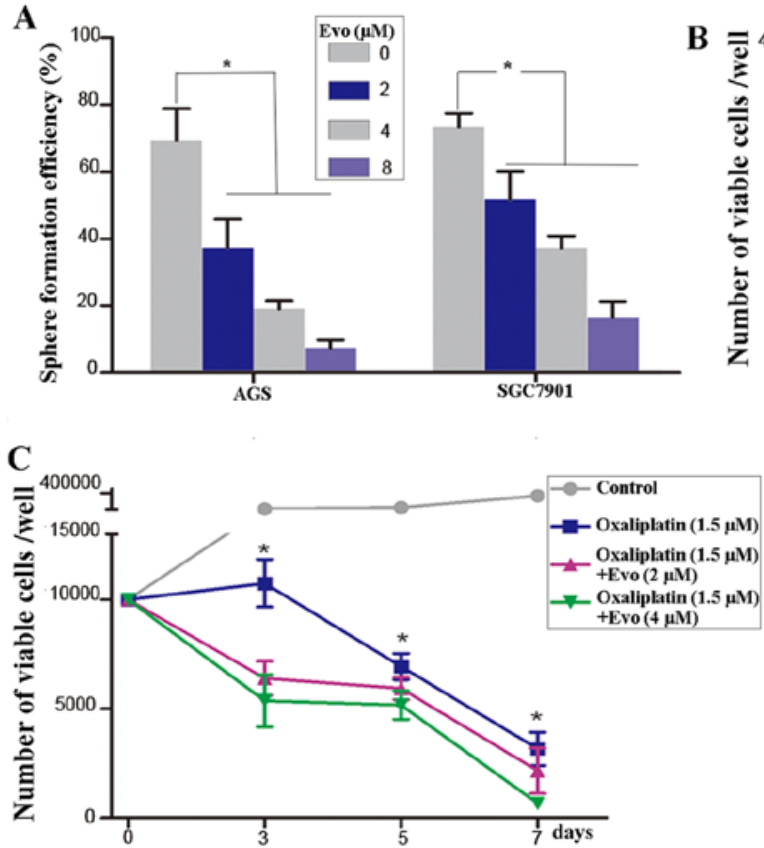

E

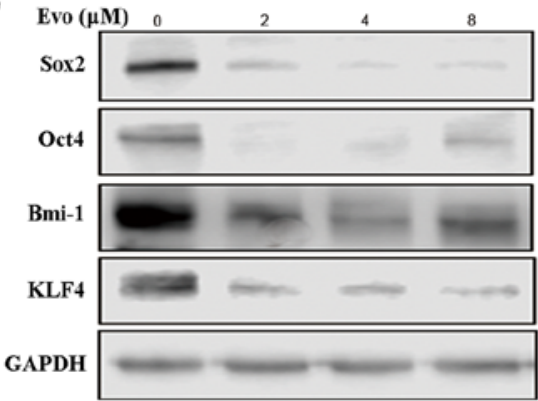

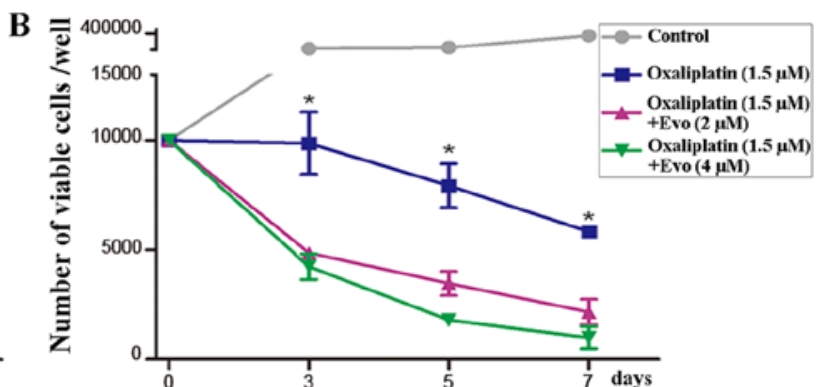

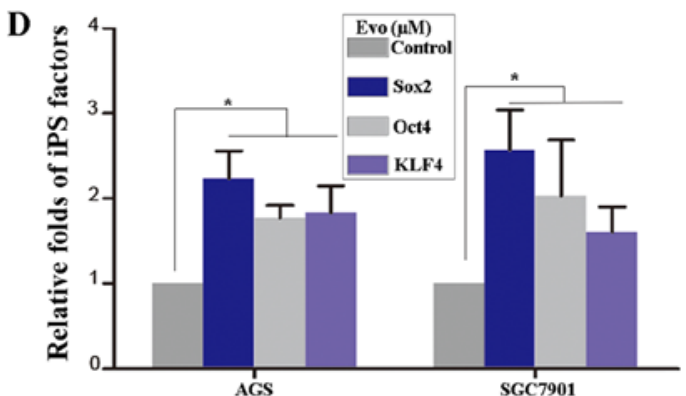

F

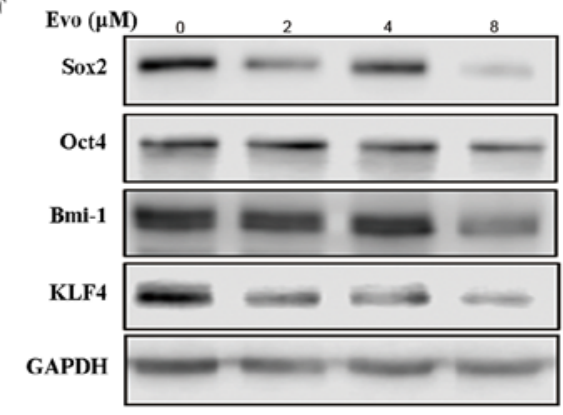

Figure 5. Evo represses the self-renewal of gastric cancel stem cells. (A) Sphere formation efficiency was assessed in AGS and SGC7901 cells with or without treatment of Evo. Results are presented as mean \pm SD of three independent experiments. (" $\mathrm{P}<0.05, \mathrm{n}=9$, ANOVA). Total number of (B) viable AGS and (C) SGC7901 cells in medium containing Evo and oxaliplatin or oxaliplatin only at the indicated concentration were counted. ("P $<0.05$, ANOVA). (D) Reverse transcription-quantitative polymerase chain reaction was performed to detect the mRNA level of iPS factors of gastric cancer cells treated with or without Evo $(2 \mu \mathrm{M})$ for $48 \mathrm{~h}$. Values represent mean $\pm \mathrm{SD}$. ("P<0.05, ANOVA). The expression of iPS factors of (E) AGS and (F) SGC7901 was measured using western blot analysis. SD, standard deviation; ANOVA, analysis of variance; iPS, induced-pluripotent stem.

significantly elevated in GCSCs (Fig. 7A) and a $\beta$-catenin inhibitor and inhibition of the Wnt pathway decreased sphere formation ability and stemness of GCCs (Fig. 7B). As the Wnt pathway is necessary to maintain self-renewal of GSCS, whether Evo-mediated inhibition of proliferation was via the Wnt pathway was investigated. The expression levels of $\beta$-catenin, c-Myc and cyclin D1 in GCSCs were all reduced by Evo in a dose-dependent manner (Fig. 7C and D). These findings implicated a role for the $\mathrm{Wnt} / \beta$-catenin signaling pathway in Evo-induced regulation of GCSC self-renewal.

\section{Discussion}

Although CSCs represent only a minor subpopulation of cells within a tumor, they are thought to have a major role in tumor initiation and resistance to chemotherapy. CSCs have been identified in solid tumors in lung, breast and colon cancer. These cells represent a promising target for the development of novel anticancer drugs that can inhibit all CSCs in a tumor and prevent recurrence (21). Traditional treatments, which receive significant attention in the field of cancer research, often fail, demonstrating the necessity for novel therapeutic options for the treatment of GC. For targeted eradication of GCSCs, several novel strategies have been proposed, including induction of apoptosis in the tumor, manipulation of GCSC cell surface molecules, development of monoclonal antibodies, modulation of the GCSC microenvironment and inhibition of GCSC pathways (3). Treatment with Evo induced apoptosis and inhibited the stemness of GCSCs by inhibiting the Wnt pathway in the present study, thereby providing a therapeutic approach to inhibit GCSC-targeted pathways.

Evo is widely used in Chinese herbal medicine, with various effects. In vitro studies showed that Evo could induce apoptosis and autophagy in GCCs $(20,22)$. However, whether Evo inhibits proliferation of GC through induction of apoptosis in GCSCs was unclear. In the present study, Evo inhibited the proliferation of GCSCs, as shown by the MTS assay and Evo induced apoptosis of GCSCs, as apparent from flow cytometry. In addition, Evo $(8 \mu \mathrm{M})$ reduced the expression of an antiapoptotic Bcl-2 family member (such as Bcl-2) in a dose-dependent 


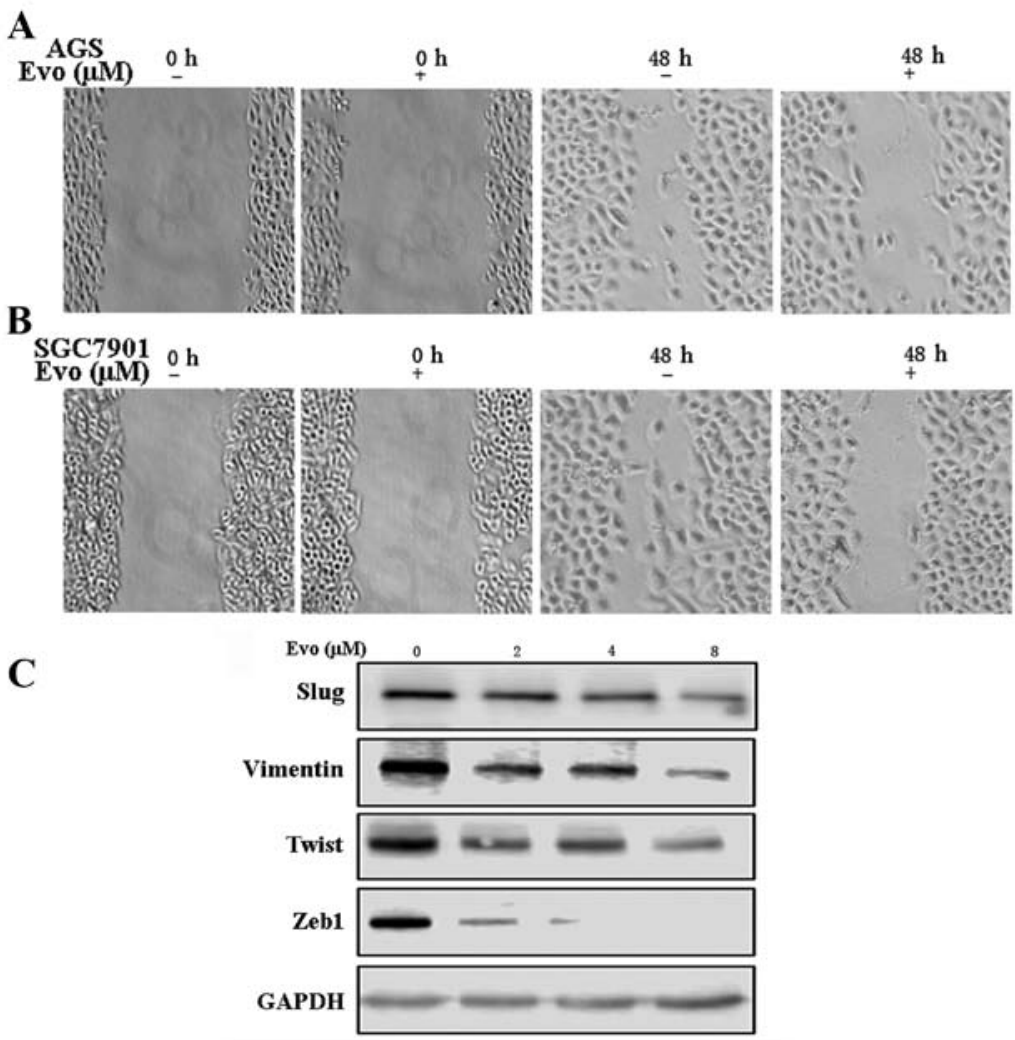

Figure 6. Evo represses the epithelial-to-mesenchymal transition in gastric cancer cells. (A and B) Migration ability was observed using a wound-healing assay. (A) AGS and (B) SGC7901 cells were treated with or without Evo, and wounds generated were observed and captured. (C) Western blot analysis was performed to detect the expression of Slug, Twist, Zeb1 and vimentin.
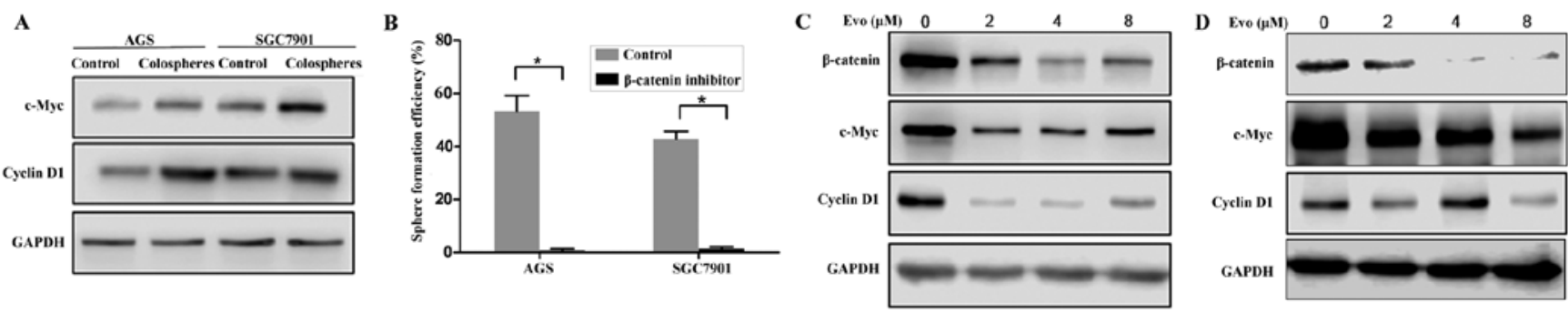

Figure 7. Evo inhibits the stem cell properties of GCSCs through the Wnt/B-catenin signaling pathway. (A) Wnt pathway activity was detected in GCSCs. Western blot analysis was performed to detect the expression of c-Myc and cyclin D1 in GCSCs and GCCs. (B) Sphere formation efficiency was decreased by the inhibition of Wnt activity in gastric cancer cells. GCCs were seeded in low adhesion plates with serum-free medium, which were subsequently supplemented with $2.5 \mu \mathrm{M} \beta$-catenin inhibitor; the total number of colospheres was counted after 7 days. Values represent the mean \pm standard deviation of three independent experiments. ( $\mathrm{P}<0.05, \mathrm{n}=9$, analysis of variance). (C and D) The downstream effector molecules of the Wnt pathway in GCSCs enriched from (C) AGS and (D) SGC7901 cells were detected by western blot analysis after treatment with Evo for $48 \mathrm{~h}$. GCSCs, gastric cancel stem cells; GCCs, gastric cancer cells.

manner, whereas it significantly elevated proapoptotic Bcl-2 family member proteins (such as Bax), resulting in upregulation of the $\mathrm{Bax} / \mathrm{Bcl}-2$ ratio. Thus, it is reasonable to suggest that Evo promoted apoptosis of GCSCs. Additional studies are necessary to further delineate which pathways are involved in Evo-induced apoptosis, the intrinsic or extrinsic apoptotic pathways or both. In addition to the effects of Evo on apoptosis, Evo reduced the spheres formation ability, inhibited the drug resistance of oxaliplatin, and decreased the expression of iPS factors of GCCs, revealing that Evo also inhibited the self-renewal of GCSCs.

The Wnt pathway is a critical signaling axis that regulates developmental processes in the embryo and maintains the self-renewal and differentiation of stem cells (23). Inhibition of $\beta$-catenin was shown to decrease the ability of gastric cancer cell sphere formation. In addition, Evo downregulated the expression of $\beta$-catenin, cyclin D1 and c-Myc in GCSCs in a dose-dependent manner, suggesting that Evo inhibited the self-renewal of GCSCs by regulating the Wnt pathway.

The EMT is a fundamental process that is critical for early embryo patterning during gastrulation, wound healing and fibrotic disease (24). Aberrant induction of the EMT has been shown to have a crucial role in the origination, invasion and metastasis of various tumors, including gastric cancer $(25,26)$. Additionally, EMT endows cellular plasticity and the properties of stemness in mammary epithelial cells (27). The EMT has strong link with iPS factors, such as Oct4 and Sox2 factors $(28,29)$, suggesting that the EMT may give rise to 
cancer stem cells. EMT is also correlated with activity in the Wnt pathway. In particular, Slug, regulated by canonical Wnt signaling, is critically important in EMT-induction of transcription factors (30). In a wound-healing assay, Evo significantly inhibited the migratory properties of GCCs. This phenomenon was further corroborated by detecting the expression of Slug, Twist, vimentin and Zeb1 in SGC7901 cells treated with Evo. Notably, these were not detected in AGS cells. The present findings suggest that Evo could inhibit the expression of EMT factors in GCCs, perhaps leading to a decrease in stemness of GCCs.

In conclusion, Evo significantly inhibited the proliferation and self-renewal of GCSCs and decreased the expression of EMT factors via downregulation of the Wnt pathway. Given the role of the Wnt pathway in carcinogenesis in GCSCs, Evo may be a potential novel antitumor agent for the treatment of gastric cancer. However, bioavailability of Evo is low, due to its poor water solubility, thereby limiting its anticancer efficacy clinically. Future studies should aim to evaluate the effects of Evo in vivo and to further develop its use as an anticancer drug.

\section{Acknowledgements}

The authors would like to thank Ms. Theresa Fu for English language editing. The present study was supported by the Zhejiang Provincial Traditional Chinese Medicine Science Research Foundation (no. 2015ZA010) and the Zhejiang Provincial Medical and Health science Foundation (no. 2015KYB020).

\section{References}

1. Ferlay J, Shin HR, Bray F, Forman D, Mathers C and Parkin DM: Estimates of worldwide burden of cancer in 2008: GLOBOCAN 2008. Int J Cancer 127: 2893-2917, 2010.

2. Fernández-Fernández FJ and Sesma P: Gastric cancer. Lancet 374: 1594, author reply 1594-1595, 2009.

3. Singh SR: Gastric cancer stem cells: A novel therapeutic target. Cancer Lett 338: 110-119, 2013.

4. Stojnev S, Krstic M, Ristic-Petrovic A, Stefanovic V and Hattori T: Gastric cancer stem cells: Therapeutic targets. Gastric Cancer 17: 13-25, 2014.

5. Takaishi S, Okumura T, Tu S, Wang SS, Shibata W, Vigneshwaran R, Gordon SA, Shimada $Y$ and Wang TC: Identification of gastric cancer stem cells using the cell surface marker CD44. Stem Cells 27: 1006-1020, 2009.

6. Yang L, Ping YF, Yu X, Qian F, Guo ZJ, Qian C, Cui YH and Bian XW: Gastric cancer stem-like cells possess higher capability of invasion and metastasis in association with a mesenchymal transition phenotype. Cancer Lett 310: 46-52, 2011.

7. Ryu HS, Park J, Kim HH, Kim WH and Lee HS: Combination of epithelial-mesenchymal transition and cancer stem cell-like phenotypes has independent prognostic value in gastric cancer. Hum Pathol 43: 520-528, 2012.

8. Han ME and Oh SO: Gastric stem cells and gastric cancer stem cells. Anat Cell Biol 46: 8-18, 2013.

9. Mishra L, Shetty K, Tang Y, Stuart A and Byers SW: The role of TGF-beta and Wnt signaling in gastrointestinal stem cells and cancer. Oncogene 24: 5775-5789, 2005.

10. Clevers H, Loh KM and Nusse R: Stem cell signaling. An integral program for tissue renewal and regeneration: Wnt signaling and stem cell control. Science 346: 1248012, 2014.

11. Mo XM, Li HH, Liu M and Li YT: Downregulation of GSK3 $\beta$ by miR-544a to maintain self-renewal ability of lung caner stem cells. Oncol Lett 8: 1731-1734, 2014.
12. Zhao Z, Lu P, Zhang H, Xu H, Gao N, Li M and Liu C: Nestin positively regulates the $\mathrm{Wnt} / \beta$-catenin pathway and the proliferation, survival and invasiveness of breast cancer stem cells. Breast Cancer Res 16: 408, 2014.

13. Watanabe K, Biesinger J, Salmans ML, Roberts BS, Arthur WT, Cleary M, Andersen B, Xie X and Dai X: Integrative ChIP-seq/microarray analysis identifies a CTNNB1 target signature enriched in intestinal stem cells and colon cancer. PLoS One 9: e92317, 2014.

14. Mao J, Fan S, Ma W, Fan P, Wang B, Zhang J, Wang H, Tang B, Zhang Q, Yu X, et al: Roles of Wnt $/ \beta$-catenin signaling in the gastric cancer stem cells proliferation and salinomycin treatment. Cell Death Dis 5: e1039, 2014.

15. Cai $C$ and Zhu $X$ : The Wnt $/ \beta$-catenin pathway regulates self-renewal of cancer stem-like cells in human gastric cancer. Mol Med Rep 5: 1191-1196, 2012.

16. Kobayashi Y: The nociceptive and anti-nociceptive effects of evodiamine from fruits of Evodia rutaecarpa in mice. Planta Med 69: 425-428, 2003.

17. Wang S, Wang L, Shi Z, Zhong Z, Chen M and Wang Y: Evodiamine synergizes with doxorubicin in the treatment of chemoresistant human breast cancer without inhibiting P-glycoprotein. PLoS One 9: e97512, 2014.

18. Chien CC, Wu MS, Shen SC, Ko CH, Chen CH, Yang LL and Chen YC: Activation of JNK contributes to evodiamine-induced apoptosis and $\mathrm{G} 2 / \mathrm{M}$ arrest in human colorectal carcinoma cells: A structure-activity study of evodiamine. PLoS One 9: e99729, 2014.

19. Wei J, Li Z and Yuan F: Evodiamine might inhibit TGF-beta1-induced epithelial-mesenchymal transition in NRK52E cells via Smad and PPAR-gamma pathway. Cell Biol Int 38: 875-880, 2014.

20. Yang L, Liu X, Wu D, Zhang M, Ran G, Bi Y and Huang H: Growth inhibition and induction of apoptosis in SGC-7901 human gastric cancer cells by evodiamine. Mol Med Rep 9: $1147-1152,2014$

21. Visvader JE and Lindeman GJ: Cancer stem cells: Current status and evolving complexities. Cell Stem Cell 10: 717-728, 2012.

22. Rasul A, Yu B, Zhong L, Khan M, Yang H and Ma T: Cytotoxic effect of evodiamine in SGC-7901 human gastric adenocarcinoma cells via simultaneous induction of apoptosis and autophagy. Oncol Rep 27: 1481-1487, 2012.

23. Van Camp JK, Beckers S, Zegers D and Van Hul W: Wnt signaling and the control of human stem cell fate. Stem Cell Rev 10: 207-229, 2014.

24. Lee JM, Dedhar S, Kalluri R and Thompson EW: The epithelial-mesenchymal transition: New insights in signaling, development, and disease. J Cell Biol 172: 973-981, 2006.

25. Montemayor-Garcia C, Hardin H, Guo Z, Larrain C, Buehler D, Asioli S, Chen H and Lloyd RV: The role of epithelial mesenchymal transition markers in thyroid carcinoma progression. Endocr Pathol 24: 206-212, 2013.

26. Zhao L, Li W, Zang W, Liu Z, Xu X, Yu H, Yang Q and Jia J: JMJD2B promotes epithelial-mesenchymal transition by cooperating with $\beta$-catenin and enhances gastric cancer metastasis. Clin Cancer Res 19: 6419-6429, 2013.

27. Mani SA, Guo W, Liao MJ, Eaton EN, Ayyanan A, Zhou AY, Brooks M, Reinhard F, Zhang CC, Shipitsin M, et al: The epithelial-mesenchymal transition generates cells with properties of stem cells. Cell 133: 704-715, 2008.

28. Luo W, Li S, Peng B, Ye Y, Deng X and Yao K: Embryonic stem cells markers SOX2, OCT4 and Nanog expression and their correlations with epithelial-mesenchymal transition in nasopharyngeal carcinoma. PLoS One 8: e56324, 2013.

29. Chiou SH, Wang ML, Chou YT, Chen CJ, Hong CF, Hsieh WJ, Chang HT, Chen YS, Lin TW, Hsu HS, et al: Coexpression of Oct 4 and Nanog enhances malignancy in lung adenocarcinoma by inducing cancer stem cell-like properties and epithelial-mesenchymal transdifferentiation. Cancer Res 70: 10433-10444, 2010.

30. Wu ZQ, Li XY, Hu CY, Ford M, Kleer CG and Weiss SJ: Canonical Wnt signaling regulates Slug activity and links epithelial-mesenchymal transition with epigenetic Breast Cancer 1, Early Onset (BRCA1) repression. Proc Natl Acad Sci USA 109: 16654-16659, 2012. 